\title{
Though Miles Apart
}

\section{Soft Tangible Interface that Evokes Nostalgic Memories}

\section{Annie Sungkajun \& Jinsil Seo}

Illinois State University, Normal, IL | Texas A\&M University, College Station, TX

\begin{abstract}
"We comfort ourselves by reliving memories of protection. Something closed must retain our memories, while leaving them their original value as images."
\end{abstract}

- Gaston Bachelard, Poetics of Space

\section{INTRODUCTION}

Though Miles Apart is an interactive installation that invites audience to physically touch soft sensors to engage with five culturally diverse memories from five individuals. When an audience member approaches to the installation, five soft pebble-like objects glow to attract their attention. As the participant kneels down to touch one of the glowing sensors, a designated video narrative is projected onto the pond-like surface and will play once it is done or until the participant plays another video.

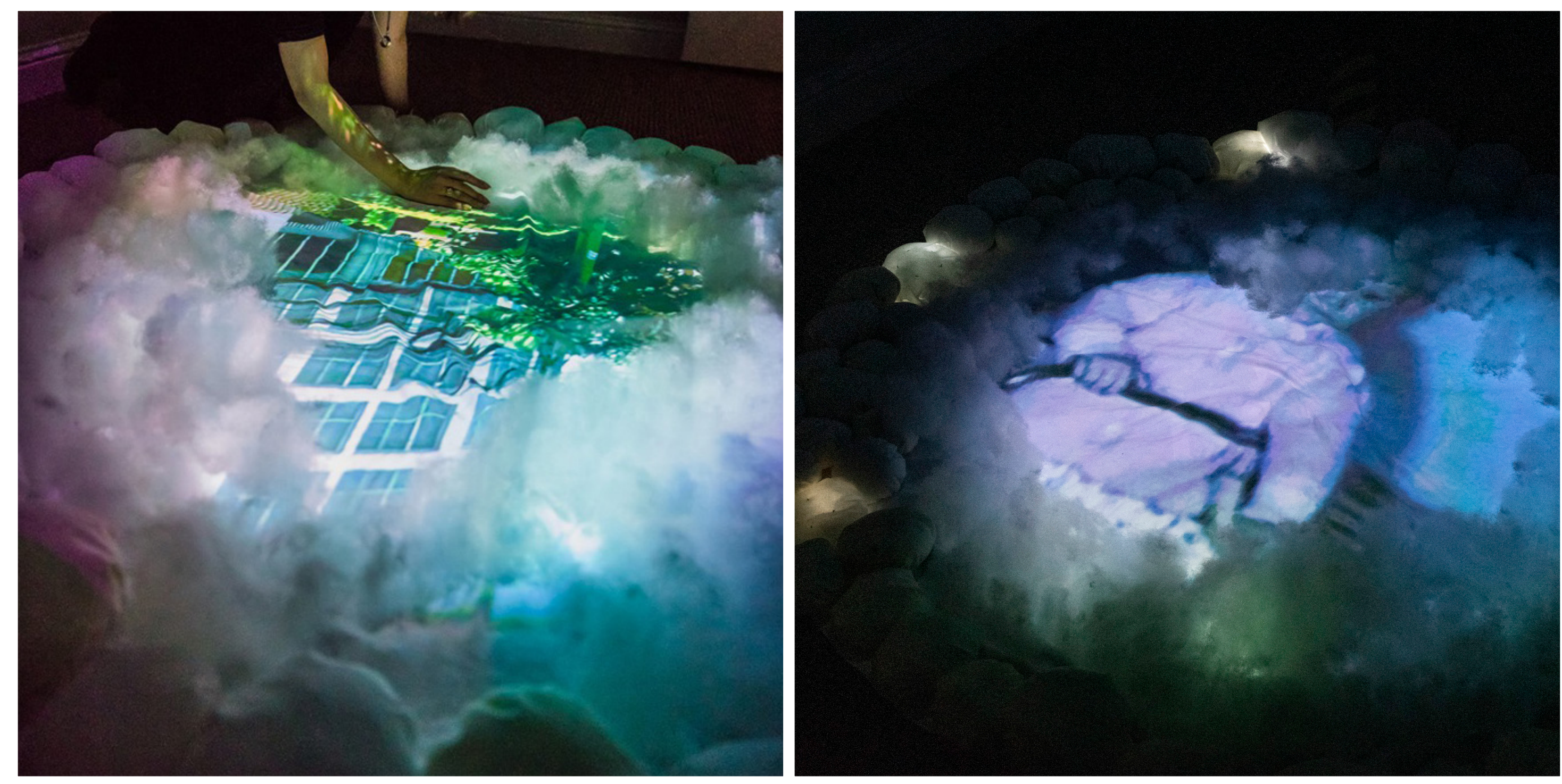

Figure 1 (left) \& 2 (right): Though Miles Apart installation

\section{INSTALLATION}

As visitors approach the dimly lit space, the first thing they see are five glowing soft, areas of a circle of pebble-like forms. Within the middle of the soft puffs plays an initial video of ripples expanding from the lit, sensing areas. Once a participant touches the sensing area, a short thirty seconds video of one of the five different narratives will play. A translated version of an excerpt from Shuidiao Getou's melody plays in accordance to the native origin of the person. Once played, the video fades back into its initial state, waiting for a participant to play another video.

The installation's design is reminiscent of a circular pond, with soft pebble-like forms outlining the border. Within the space, polyfill obscures parts of the projected image and acts as a 'fog' in order to cloud the memory shown. It is encouraged for participants to push away at the fog, to gain clarity of the imagery. Calling back to the inspiration's notion of the moon, the glow of the projection mimics the glow of a moon on a pond's surface.

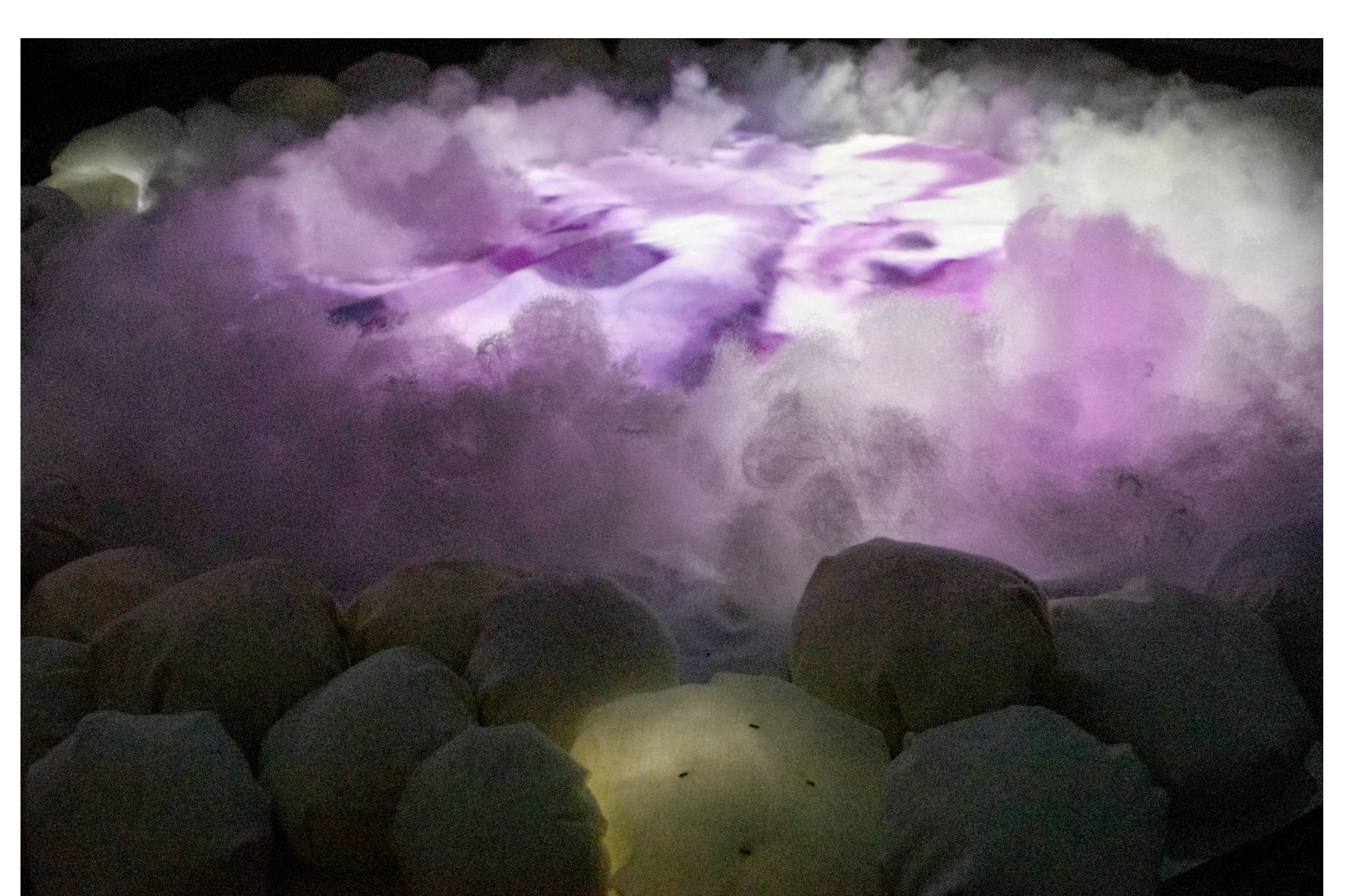

Figure 3: Close-up of installation; polyfill acts as a cloud to 'fog' memory.

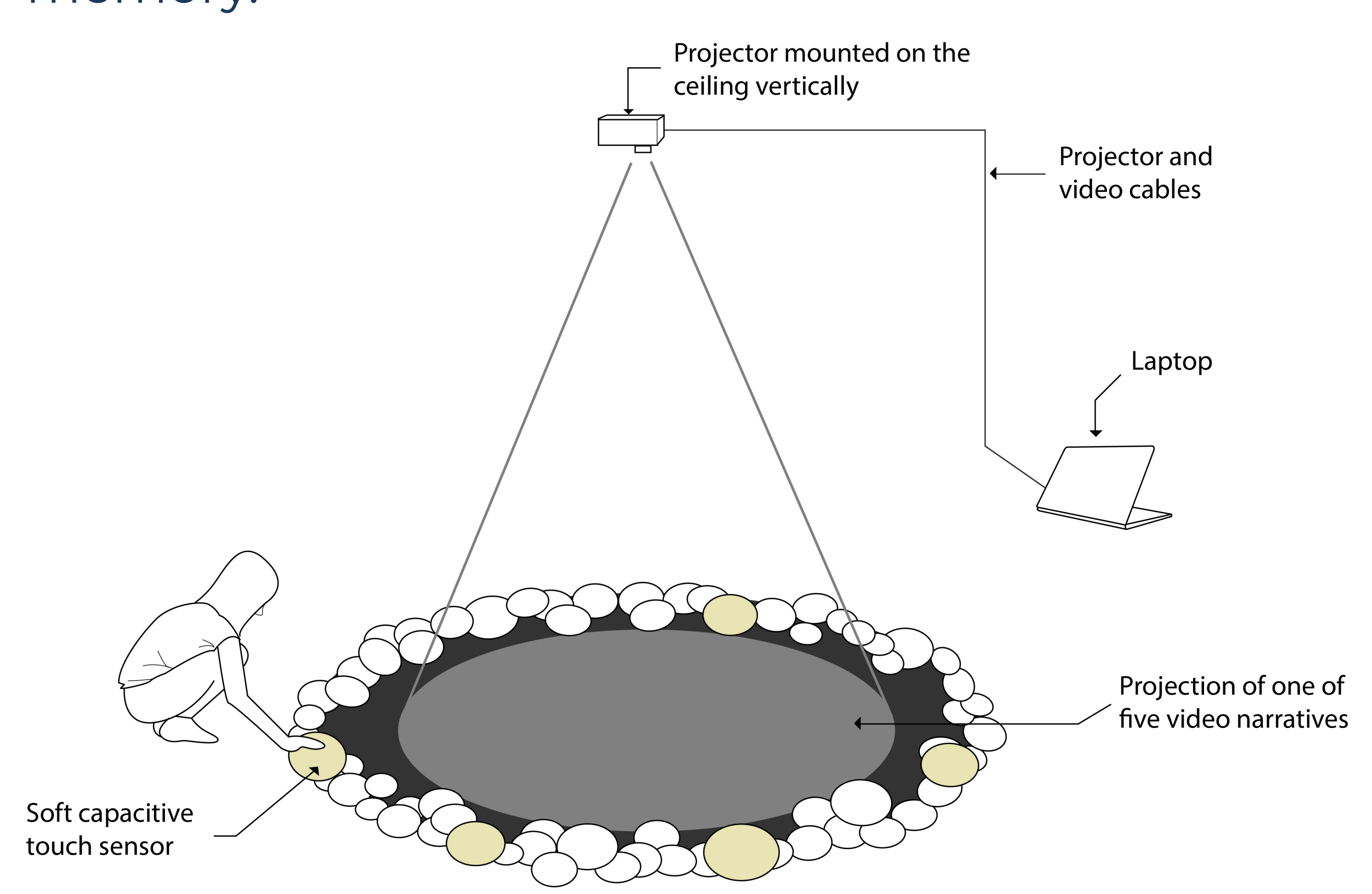

Figure 4: Though Miles Apart diagram

\section{SOFT TANGIBLE CIRCUIT}

Among the soft, pebble-like forms along the outer edge, there are five glowing conductive sensing areas. These areas produce a fading pattern to show their inactivity and will only glow solidly when they are grasped. This is done through soft circuitry, which are electrical circuits made from textiles. These sensing areas were made as capacitive sensors, using conductive thread and an Arduino microcontroller. LEDs are embedded within the areas to act as visual feedback to the viewer.

\section{PROJECTION}

The footage used within the installation was sourced from five people of different cultures - Korean, Arabic, Thai, Peruvian, and American - experiencing disconnectedness amongst family members. They provided a range of footage from either childhood to the present, as memories they were willing to share. A parallax effect was applied to the photos in order to make them feel more 'alive'. The footage was then edited to create abstract imagery, in order to focus on universal elements that can be relatable to anyone. The sound is an excerpt from Shuidiao Getou's melody, translated into the different languages.

Though Miles Apart explores the act of reminiscence through projected imagery of memories from people of different cultures. While

the memories were individualized to the person that provided it, visitors of no relation are still able to extract their own nostalgia through the footage and conversation. The concept was realized as a soft, tangible interface to mimic the setting of a pond in moonlight. Reflecting Shuidiao Getou's moon and through the process of reminiscing, though we may be miles apart from the people we share memories with, we are still able to share the beauty of them together 